\title{
Utilizing Respiratory Therapists to Reduce Costs of Care
}

\author{
Ellen A Becker PhD RRT-NPS RPFT AE-C FAARC, Cheryl A Hoerr MBA RRT CPFT FAARC, \\ Kimberly S Wiles RRT CPFT, Debra L Skees MBA RRT CPFT, Corinne H Miller MLIS, and \\ Douglas S Laher MBA RRT FAARC
}

\begin{abstract}
INTRODUCTION: Changes to the reimbursement of respiratory care services over the past 26 years make it imperative that respiratory therapists (RTs) demonstrate cost savings to establish their value. Therefore, this systematic review evaluated the cost-related impacts from utilizing RTs to deliver care when compared to other care providers. METHODS: The Preferred Reporting Items for Systematic Reviews and Meta-Analyses guidelines were used to guide the search process. The study addressed articles across all age groups and care settings that compared the cost of care provided by RTs to a comparison group. Studies were excluded if they were not written in English, described care provided outside of the United States, did not provide quantitative data, or lacked a comparison group. RESULTS: A total of 4,120 articles emerged from the search process, of which 60 qualified for a full text review. Cost savings were evaluated for the 28 articles included in this review, noting the study design, the specific respiratory care practice, use of protocols, clinical setting, and age group. The most frequently studied topic was mechanical ventilation, which along with disease management represented by the most randomized, controlled trials for the study design. The clinical practice area notably absent was home care. CONCLUSIONS: Although cost comparisons across studies could not be made due to the inconsistent manner in which data were reported, evidence demonstrated that care provided by RTs yielded both direct and indirect cost reductions, which were achieved through protocol utilization, specialized expertise, and autonomous decision making. The care provided was consistent with care provided by other disciplines. It is critical for the respiratory care profession to highlight key clinical practice areas for future research, to establish uniform reporting measures for outcomes, and to foster the development of future respiratory care researchers to affirm the value that respiratory therapists add to patient care. Key words: respiratory therapy; cost-benefit analysis; cost control; health care costs [Respir Care 2018;63(1):102-117. (c) 2018 Daedalus Enterprises]
\end{abstract}

\section{Introduction}

With its beginning in 1943, the respiratory therapy profession is a relative newcomer when compared with the longer established professions of medicine and nursing. Through 1957, respiratory therapists (RTs) received onthe-job training ${ }^{1}$ and primarily provided oxygen therapy to

Dr Becker is affiliated with the Department of Cardiopulmonary Sciences, Rush University Medical Center, Chicago, IL. Ms Hoerr is affiliated with the Department of Respiratory and Sleep Services; Phelps County Regional Medical Center, Rolla, MO. Ms Wiles is affiliated with Allegheny Health Network Home Medical Equipment, Ford City, PA. Ms Skees is affiliated with the Department of Respiratory Services, Mercy Hospital-Allina Health, Coon Rapids, MN. At the time of this study, Ms. Miller, MLIS was affiliated with Rush University Medical Center, postsurgical patients. New technologies for positive-pressure ventilators and nebulizers were developed in the 1960s. In the 1970s and 1980s, respiratory therapy services ex-

Chicago, IL and is now affiliated with Galter Health Sciences Library, Fein-
berg School of Medicine, Northwestern University, Chicago, IL. Mr Laher
is affiliated with the American Association for Respiratory Care, Irving, TX.

The authors report that they each have served or are now serving on the AARC Board of Directors; Mr Laher is an AARC Associate Executive Director.

Correspondence: Ellen A Becker PhD RRT-NPS RPFT AE-C FAARC, Department of Cardiopulmonary Sciences, Rush University Medical Center, 600 S. Paulina Street, AAC Suite 750, Chicago, IL 60612. E-mail: ellen_becker@rush.edu.

DOI: $10.4187 /$ respcare. 05808 
panded into the areas of laboratory-based diagnostics, airway care, bronchial hygiene, aerosol therapy, and ventilator management. ${ }^{1,2}$

Intermittent positive-pressure breathing, a prominent early therapy, was indiscriminately used by ordering prescribers when there was little evidence of effectiveness. Published research from as early as 1974 highlighted the misuse of this therapy. In the same year, the first Conference on the Scientific Basis for Respiratory Therapy reported on the abuse and actively discouraged the use of intermittent positive-pressure breathing. ${ }^{3}$ This experience highlighted the need for greater emphasis on a scientific basis for all respiratory therapies. In the 1990s, the American Association for Respiratory Care (AARC) developed clinical practice guidelines to highlight the science behind respiratory therapy and enhance the credibility of its practices. $^{2}$ These guidelines have since transitioned from expert panel opinion to evidence-based guidelines utilizing peer-reviewed scientific research and strength of evidence for making clinical recommendations. Incentive spirometry, ${ }^{4}$ airway clearance therapies, ${ }^{5}$ and pharmacologic airway-clearance therapies ${ }^{6}$ are three recent examples of therapies commonly provided by RTs that lack clinical benefit for routine use.

\section{Description of Issues}

Throughout most of its existence, the respiratory care department functioned under a fee-for-service model of payment in which the health care provider is paid a fee for each particular service rendered. ${ }^{7}$ Respiratory care departments were revenue centers, and charges for services provided contributed to the organizations' bottom lines. Physicians placed an order for therapy, the RT provided the therapy as ordered, the hospital charged the patient each time the therapy was provided, and the patient or insurance company paid the hospital bill. Treatment volume was the primary driver of the respiratory care department budget, and treatment outcomes were not frequently reported. ${ }^{8}$

The era of diagnosis-related groups had a significant impact on the respiratory care profession's status as a revenue generator for health care organizations. By the early 1980s, it was obvious that the cost of health care in the United States was becoming unsustainable. Annual health spending between 1966 and 1982 averaged 13\% growth while the average annual GDP growth was $9.2 \% .9^{9}$ The diagnosis-related group program was implemented to bundle in-patient treatment into payment groups and capitate Medicare reimbursement based upon diagnosis. Unnecessary therapy volume became a costly detriment to the bottom line of health care organizations, and respiratory care departments scrambled to shift from volume-focused care to a system based on the appropriateness of the delivered therapy. Therapist-driven protocols emerged in the mid1980s in response to these changes. ${ }^{10}$

Health care institutions now must survive the transition from the diagnosis-related group model of reimbursement to a coordinated-care pay-for-performance model. ${ }^{11,12} \mathrm{As}$ evidence of this new approach, the Institute for Healthcare Improvement, an influential force in health and health care improvement, is committed to redesigning health care into a system without errors, waste, delay, and unsustainable costs. The Institute for Healthcare Improvement has expanded its framework to embrace the Triple Aim, a framework for optimizing health system performance by simultaneously focusing on the health of a population, the experience of care for individuals within that population, and the per-capita cost of providing that care. ${ }^{13}$ As a result, the RT's contribution is beginning to shift its focus from single-occurrence treatment to long-term disease prevention and disease management for patients at risk for respiratory complications and with chronic diseases. ${ }^{13}$

This systematic review evaluated the published literature to examine the contributions of RTs to meet the requirements of the new health care delivery environment. It addressed the findings from studies involving RTs and how the profession may have a positive impact on reducing the direct and indirect costs of care.

\section{Methods}

The Preferred Reporting Items for Systematic Reviews and Meta-Analyses (PRISMA) statement was used as a guide for this review. ${ }^{14}$ This systematic review included all quantitative research designs across all clinical practice settings.

\section{Search Strategy}

A medical librarian searched MEDLINE via PubMed 1946-, the Cumulative Index of Nursing and Allied Health Literature (CINAHL Complete) 1937-, Scopus 1823-, ProQuest Dissertations and Theses A\&I 1861-, The Gray Literature Report 1999-, and ClinicalTrials.gov.

Subject terms (when available) and key words were used to locate literature published from January 1990 to July 2016 that described how RTs impact the cost of health care. Publication type filters were utilized, when possible, to exclude letters, comments, and editorials from the results. The full search strategy for PubMed appears in Table 1. Additionally, we searched reference lists of included studies and hand-searched the last 10 years of the journals Chest and Respiratory CARE, which identified 16 additional articles. Two of these articles were published after our stated search sampling frame. The RefWorks (ProQuest, Ann Arbor, Michigan) automatic duplicate search 
Table 1. MEDLINE/PubMed Search Strategy

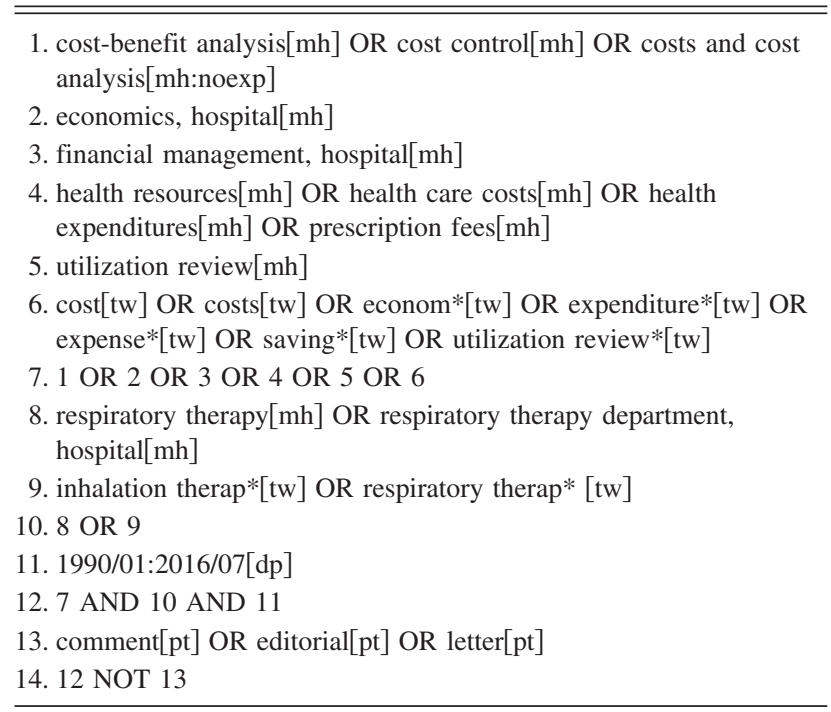

was then used to identify and remove 747 duplicate records, at which point 4,120 citations remained.

\section{Study-Inclusion Criteria}

The study included articles that considered cost-related outcomes across all age groups (neonatal, pediatric, adult, and geriatric) and all care settings (acute care [non-ICU], acute care [critical care], post-acute care) published between January 1990 and July 2016 that compared the cost of care provided by RTs to a comparison group. Studies were excluded if they were not written in English, described care provided outside of the United States, did not provide quantitative data, or lacked a comparison group.

\section{Study Procedures}

A title and abstract review was conducted by 1 of the 5 authors for each of the 4,120 identified articles. Abstracts that did not meet inclusion criteria or met exclusion criteria were removed from the review, leaving a total of 60 articles for full text review.

For the remaining 60 articles, 2 authors independently extracted the type of study design, population, intervention, comparator, and outcomes. The authors used a taxonomy for observational study designs to classify study types. ${ }^{15}$ Data for the population included the health care setting, patient population, and number of participants. For the intervention, we identified the type of health care provider conducting the intervention, the type of intervention, and comparison groups. Reduced cost of care was the primary outcome variable. The secondary outcome variables had implied cost savings and included variables such as length of stay, productivity, utilization, readmission, decreased therapies, and ventilator days. After data extraction, each review author led a discussion of analysis with the entire research team to resolve disagreements and reach consensus on research design, methodology, and outcomes. A total of 28 articles remained that met the review criteria.

\section{Measure of Treatment Effect}

The treatment effect in this study is the "value" of the RT and how therapists impact the cost of delivering care. When comparing RTs against other lower-paid health care providers, the treatment effect must not only have been positive, but must have exceeded the difference in care delivery between the two providers. When comparing RTs against themselves (protocol vs no protocol), any positive treatment effect was acceptable. When comparing RTs against physicians and nurses (higher-paid caregivers), a neutral treatment effect was acceptable so long as there were no detrimental clinical differences in outcomes.

\section{Unit of Analysis}

Financial savings (cost of delivering care) were the unit of analysis in each study. Measurements in dollars collected from each study were analyzed. For those studies that did not report direct cost savings, indirect cost savings results such as length of stay, productivity, resource utilization, readmission, decreased therapies, and ventilator days were recorded, if present.

\section{Analysis}

The studies were categorized by therapeutic topic, presence or absence of respiratory care protocol, setting, and age group. For this review, "protocol" was defined as the use of an algorithm (paper-based or automated) intended to result in standardized and expedited delivery of care. Direct costs were extracted from each study and recorded using the scale reported by the study author (eg, per case, per subject, per day). Dollar values were rounded to whole numbers to enhance clarity. Mean and median measures of indirect cost savings were recorded from each study, and the corresponding statistical significance was reported.

\section{Results}

A total of 28 articles of the 60 total articles that were reviewed met our inclusion criteria. A flow diagram summarizes the study selection (Fig. 1).

All 28 studies demonstrated cost savings. The included studies covered a wide variety of respiratory care practices, which included the topics of ventilators (9); ${ }^{16-24}$ multiple therapies (7); ${ }^{25-31}$ disease management (4);32-36 invasive procedures (3), [line insertion (2) for $\operatorname{arterial}^{37}$ and internal jugu- 


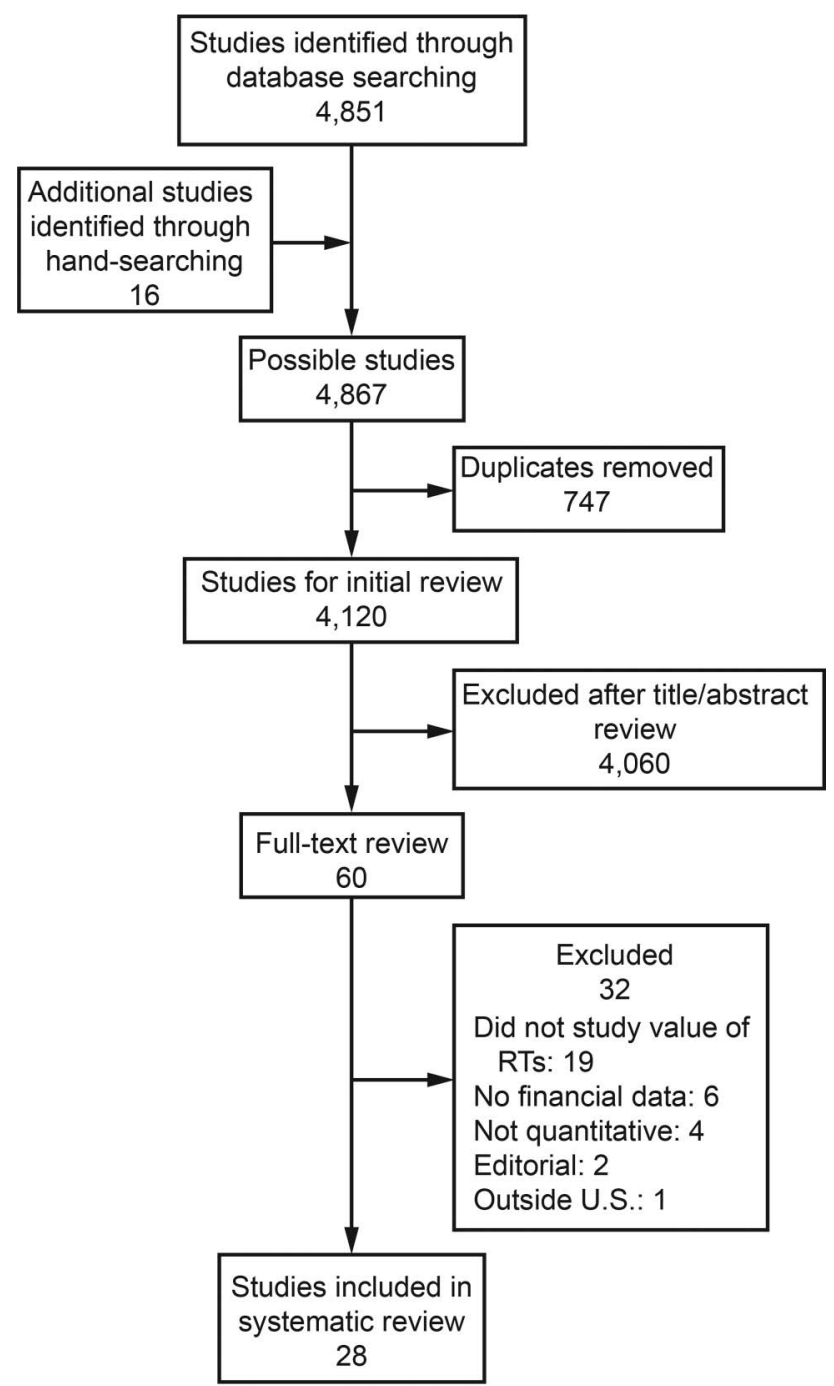

Fig. 1. Flow chart.

$\operatorname{lar}^{38}$ vessels, and intubation (1) ${ }^{39}$ ]; medication aerosol therapy (2); ${ }^{40,41}$ and oxygen (2). ${ }^{42,43}$ Sixteen of the studies (57\%) utilized protocols. Almost all studies were conducted in acute critical-care settings (11) 16-20,22-24,37-39 or acute non-ICU settings (13).21,25-31,33-35,40,41 There were 3 studies conducted in ambulatory care, ${ }^{32,36,42}$ and 1 study was conducted in a long-term care setting. ${ }^{43}$ Most studies addressed the adult population (24), with 2 each studying pediatric $^{32,34}$ and neonatal populations. ${ }^{23,39}$ These findings are summarized in Table 2.

Regarding the strength of evidence, only 6 studies utilized a randomized controlled trial, $17,20,30,33,35,36$ of which 3 addressed disease management and 2 addressed mechanical ventilation. Twelve studies used a non-concurrent cohort study design. 16,19,21-23,26,27,29,31,34,40,41 A before-andafter design was used in 4 studies, ${ }^{32,38,42,43}$ and a crosssectional design was used in 3 studies, ${ }^{25,28,37}$ as well as a controlled before-and-after study, ${ }^{24}$ a non-comparative design, ${ }^{39}$ and a retrospective cohort study. ${ }^{18}$

Given the focus of this study on cost savings, it was not surprising that the most frequently studied topic related to mechanical ventilation management/liberation protocols. Addressing an expensive service has the highest likelihood of providing the greatest cost savings. These ventilator studies frequently utilized a multidisciplinary team. Nearly one third of the studies fell into this category, and $22 \%$ of them were randomized controlled trials. All studies related to ventilation management demonstrated cost savings independent of study design. Table 3 contains the individual units of analysis and cost savings. Note that when direct cost savings were analyzed, no statistical significance was demonstrated across any of the studies. Studies revealed significant indirect cost saving such as decreased ventilator duration (7 of 9 studies), ICU length of stay, and hospital length of stay.

There was only 1 randomized controlled trial among the 6 studies that evaluated cost savings across multiple therapies. ${ }^{30}$ Compared with physician-directed respiratory care, the RTs recommended a similar number and duration of respiratory care services at a slight savings and without any increased adverse events. Care provided by RTs is less expensive and thus has the potential for cost savings. Looking at all studies that addressed multiple therapies, there were direct cost savings in $57 \%$ of them. No clear pattern for indirect cost savings emerged.

Among the 5 studies that addressed disease management, $40 \%$ reported direct cost savings, 3 of which were randomized controlled trials. Statistically significant hospital cost savings emerged from studies in both the adult and pediatric populations. The 5 studies in this category addressed 2 different conditions: asthma and COPD. Within asthma, decreased hospitalizations, hospital days, and emergency department visits were shown. All indirect costsavings measures for disease management by the RT achieved statistical significance. However, the diverse measures reported made it difficult to compare the results and make further inferences. Regarding COPD, there were mixed results. Both studies ${ }^{33,35}$ demonstrated a reduced hospital length of stay, but they differed in the impact of COPD disease management upon emergency department visits and hospital admissions.

It is noteworthy that the review did not find any qualifying studies in home care. The American Thoracic Society's position statement on home care for patients with respiratory disorders refers to the expertise of a respiratory specialist and the lack of Medicare reimbursement for RTs. ${ }^{44}$ It is presumed that the absence of funding for respiratory care services in the home may be part of the reason for the lack of documented studies as well as the reduced focus in the post-acute care setting until recently. Unique models utilizing RTs in the home to prevent re- 
Table 2. Study Attributes of Cost-Savings Articles

\begin{tabular}{|c|c|c|c|c|c|}
\hline Author & Study Type & Major Topic & Protocol & Setting & Age Group \\
\hline Ely et al $(1996)^{17}$ & Randomized controlled trial & Ventilator & Yes & Acute: critical care & Adult \\
\hline Kollef et al $(1997)^{20}$ & Randomized controlled trial & Ventilator & Yes & Acute: critical care & Adult \\
\hline Kelleghan et al $(1993)^{19}$ & Non-concurrent cohort study & Ventilator & No & Acute: critical care & Adult \\
\hline Dasgupta et al (1999) $)^{21}$ & Non-concurrent cohort study & Ventilator & No & Acute: non-ICU & Adult \\
\hline Burns et al $(2003)^{16}$ & Non-concurrent cohort study & Ventilator & Yes & Acute: critical care & Adult \\
\hline Cohen et al $(1991)^{22}$ & Non-concurrent cohort study & Ventilator & Yes & Acute: critical care & Adult \\
\hline Hermeto et al $(2009)^{23}$ & Non-concurrent cohort study & Ventilator & Yes & Acute: critical care & Neonates \\
\hline Arroliga et al $(2012)^{24}$ & Controlled before and after study & Ventilator & No & Acute: critical care & Adult \\
\hline Gupta et al $(2014)^{18}$ & Retrospective cohort study & Ventilator & Yes & Acute: critical care & Adult \\
\hline Stoller et al $(1998)^{30}$ & Randomized controlled trial & Multiple & Yes & Acute: non-ICU & Adult \\
\hline Harbrecht et al (2009) ${ }^{27}$ & Non-concurrent cohort study & Multiple & Yes & Acute: non-ICU & Adult \\
\hline Kollef et al $(2000)^{26}$ & Non-concurrent cohort study & Multiple & Yes & Acute: non-ICU & Adult \\
\hline Nyland et al (2016) ${ }^{29}$ & Non-concurrent cohort study & Multiple & Yes & Acute: non-ICU & Adult \\
\hline Stoller et al $(1996)^{31}$ & Non-concurrent cohort study & Multiple & Yes & Acute: non-ICU & Adult \\
\hline Kallam et al $(2013)^{25}$ & Cross-sectional study & Multiple & Yes & Acute: non-ICU & Adult \\
\hline Werre et al $(2015)^{28}$ & Cross-sectional study & Multiple & Yes & Acute: non-ICU & Adult \\
\hline Rice et al $(2010)^{35}$ & Randomized controlled trial & Disease management & No & Acute: non-ICU & Adult \\
\hline Shelledy et al $(2009)^{36}$ & Randomized controlled trial & Disease management & No & Ambulatory care & Adult \\
\hline Silver et al $(2017)^{33}$ & Randomized controlled trial & Disease management & No & Acute: non-ICU & Adult \\
\hline Shelledy et al $(2005)^{32}$ & Before and after study & Disease management & No & Ambulatory care & Pediatric \\
\hline Tearl et al $(2006)^{34}$ & Non-concurrent cohort study & Disease management & No & Acute: non-ICU & Pediatric \\
\hline Gronbeck et al $(1993)^{37}$ & Cross-sectional study & Invasive procedures/Line insertion & No & Acute: critical care & Adult* \\
\hline Ramirez et al $(2010)^{38}$ & Before and after study & Invasive procedures/Line insertion & No & Acute: critical care & Adult \\
\hline Noblett et al $(1995)^{39}$ & Non-comparative study & Invasive procedures/Intubation & No & Acute: critical care & Neonates \\
\hline Colice et al $(2005)^{40}$ & Non-concurrent cohort study & Aerosolized medication & Yes & Acute: non-ICU & Adult \\
\hline Tenholder et al $(1992)^{41}$ & Non-concurrent cohort study & Aerosolized medication & Yes & Acute: non-ICU & Adult* \\
\hline Chaney et al $(2002)^{42}$ & Before and after study & Oxygen & No & Ambulatory care & Adult \\
\hline Christman et al $(2006)^{43}$ & Before and after study & Oxygen & Yes & Long-term care & Adult \\
\hline
\end{tabular}

* Did not explicitly state an adult population, but adult population is assumed in reviewing the study description.

admissions are emerging, with the expectation that more qualifying studies will be documented. Preventing potentially avoidable readmissions is the primary approach by hospitals to attempt to evade Centers for Medicare and Medicaid Services penalties and minimize the negative impact to the bottom line for health care institutions. Although controversial, successful strategies can reduce readmissions that are no longer reimbursed. ${ }^{45}$

Three studies addressed procedures commonly performed by physicians: line insertion (intravenous and central line) and intubation. Two of these 3 studies had large sample sizes of $N=327^{38}$ and $N=506$, respectively. ${ }^{37}$ All 3 studies demonstrated that the success rates for RTs were as good as those for physicians without documented complications. These findings indicate that respiratory therapists possess technical expertise for invasive procedures within their scope of practice (eg, intubation, catheter insertions). Given the lower salaries of RTs compared to physicians, cost savings occur. Although reimbursement is the same to the institution independent of who provides the procedure, the cost benefit appears when the realloca- tion of providers better matches patient and organizational needs.

Two studies addressed aerosolized medication. Only the costs/admission for RT salary was statistically significant for aerosolized medication. Almost all indirect costs looked at resource-utilization variables except for decreased adverse events. In these studies, there were too little data available to draw significant conclusions on the effect of indirect cost.

Both studies addressing oxygen therapy evaluated the $\mathrm{RT}$ role in reporting cost savings, but there were no statistical analyses to support these findings. Armed with a protocol, the RT appeared to have the appropriate resource management for oxygen therapy. In $18-29 \%$ of cases, the RT implemented changes to the oxygen prescription on the basis of an assessment of need. ${ }^{43}$ The confidence in these findings, however, is low due to the absence of statistical confirmation.

Slightly more than half of the studies included in this review utilized respiratory care protocols. There were protocols related to ventilator management, aerosol therapy, 


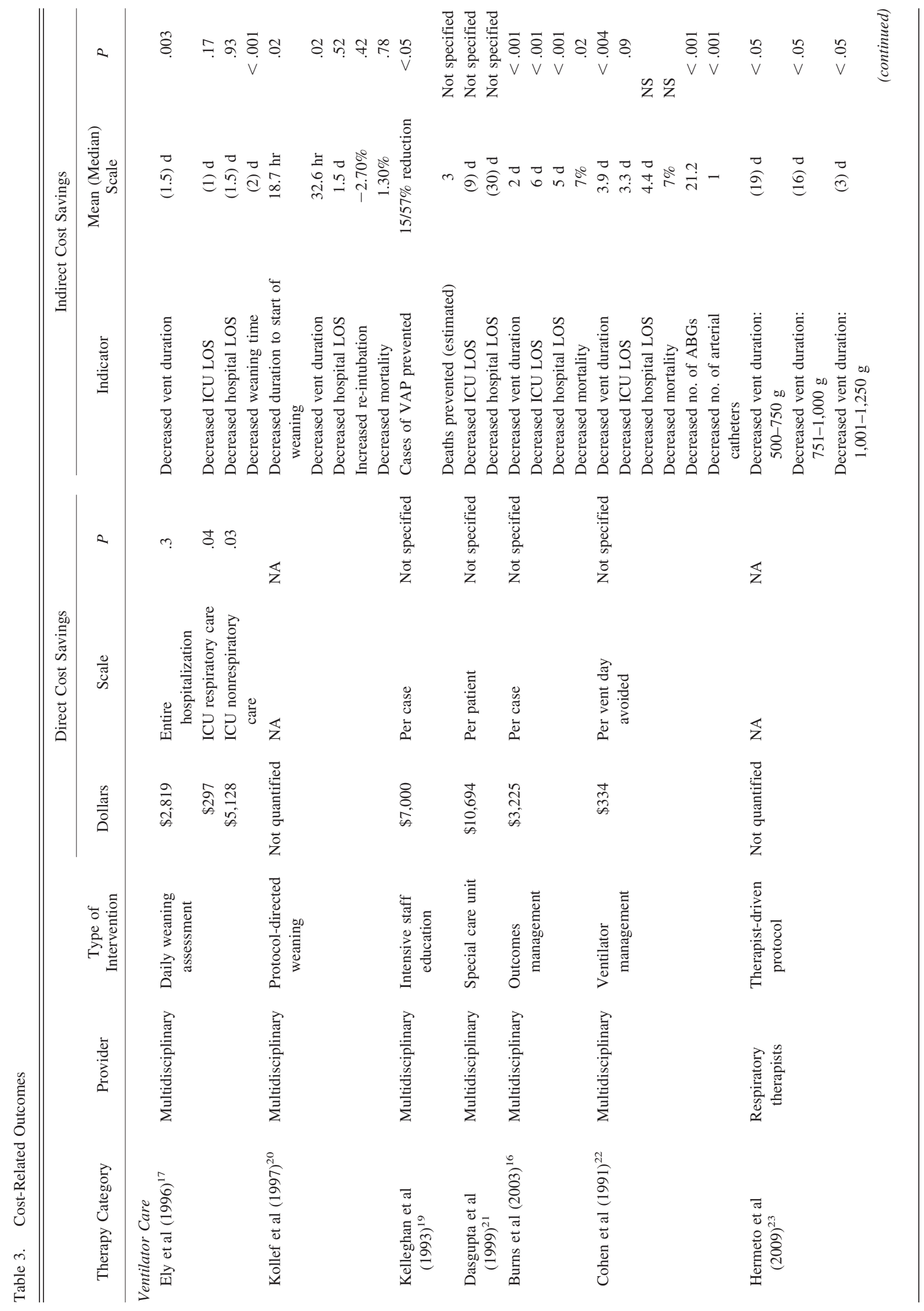


Using RTs to Reduce Cost of CARE

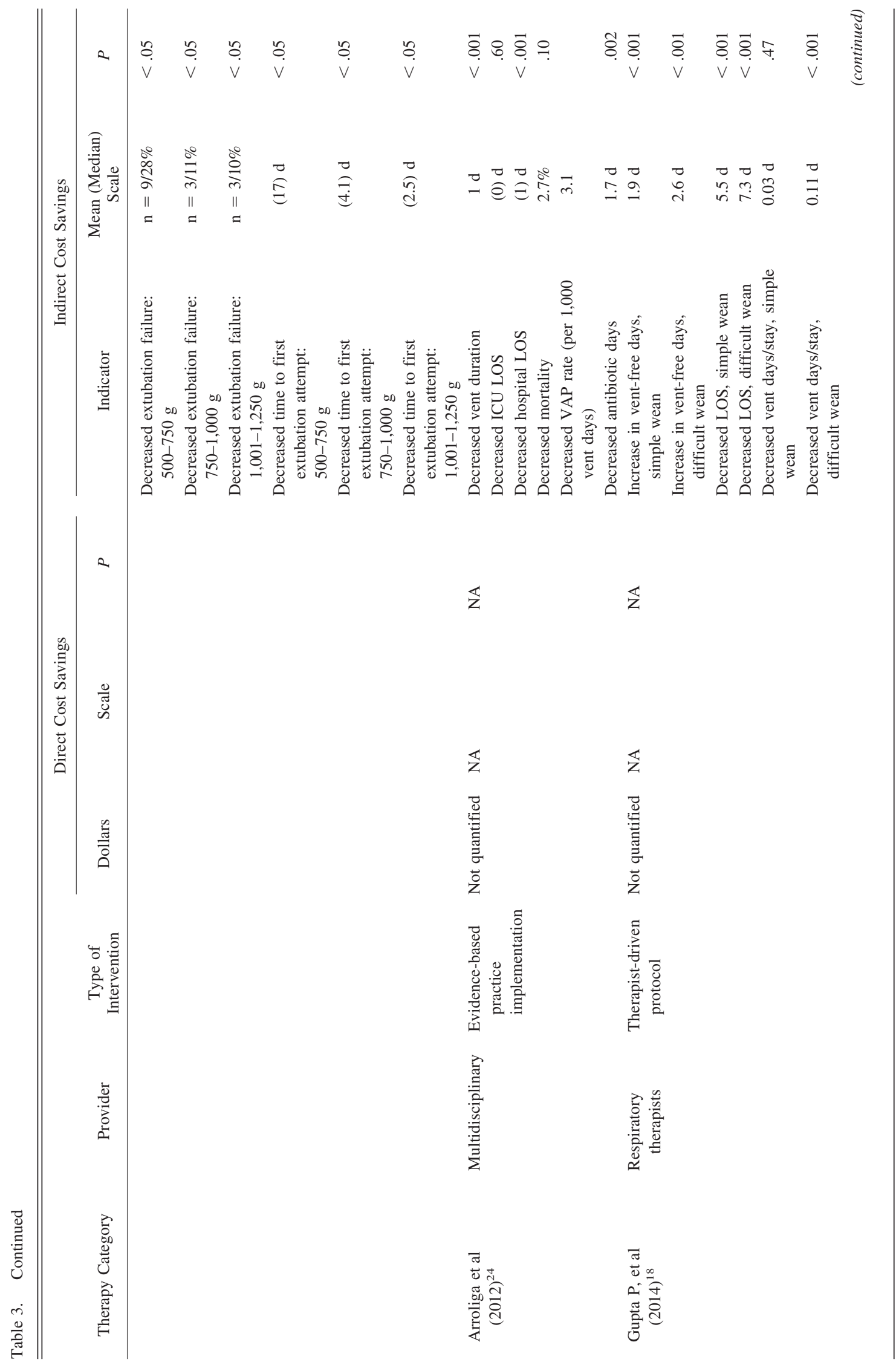




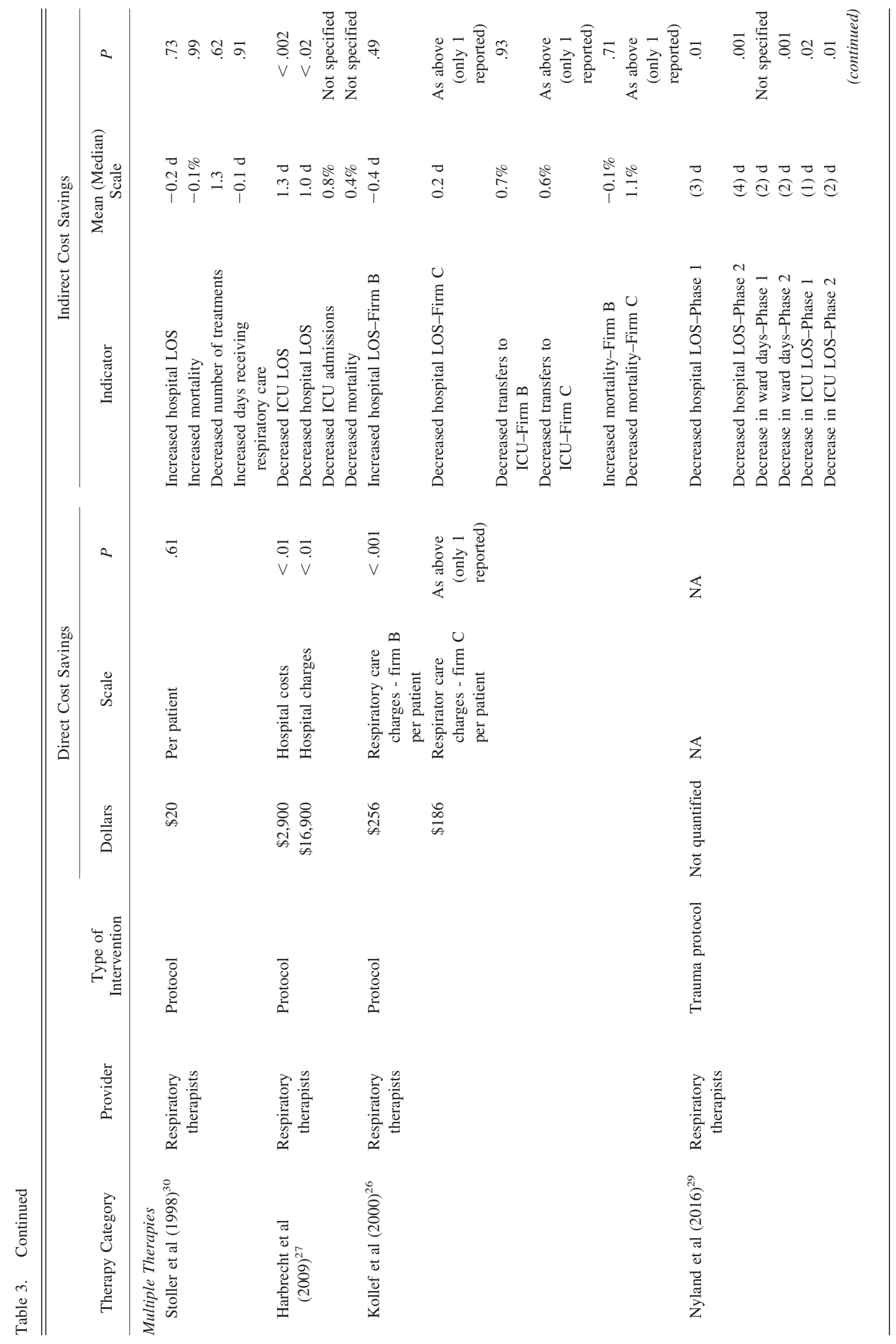


Using RTs to Reduce Cost of CARe

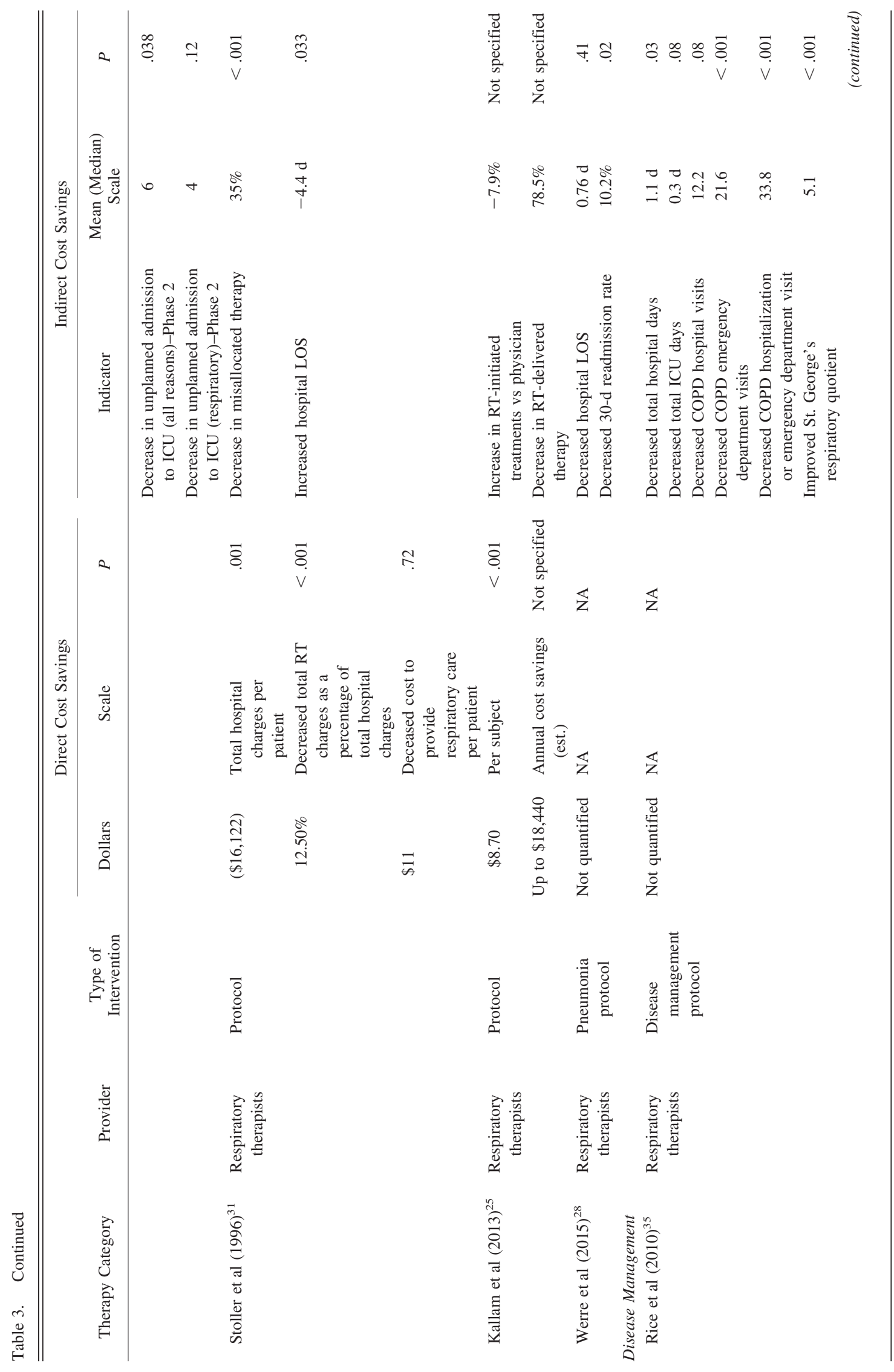


Using RTs to Reduce Cost of CARE

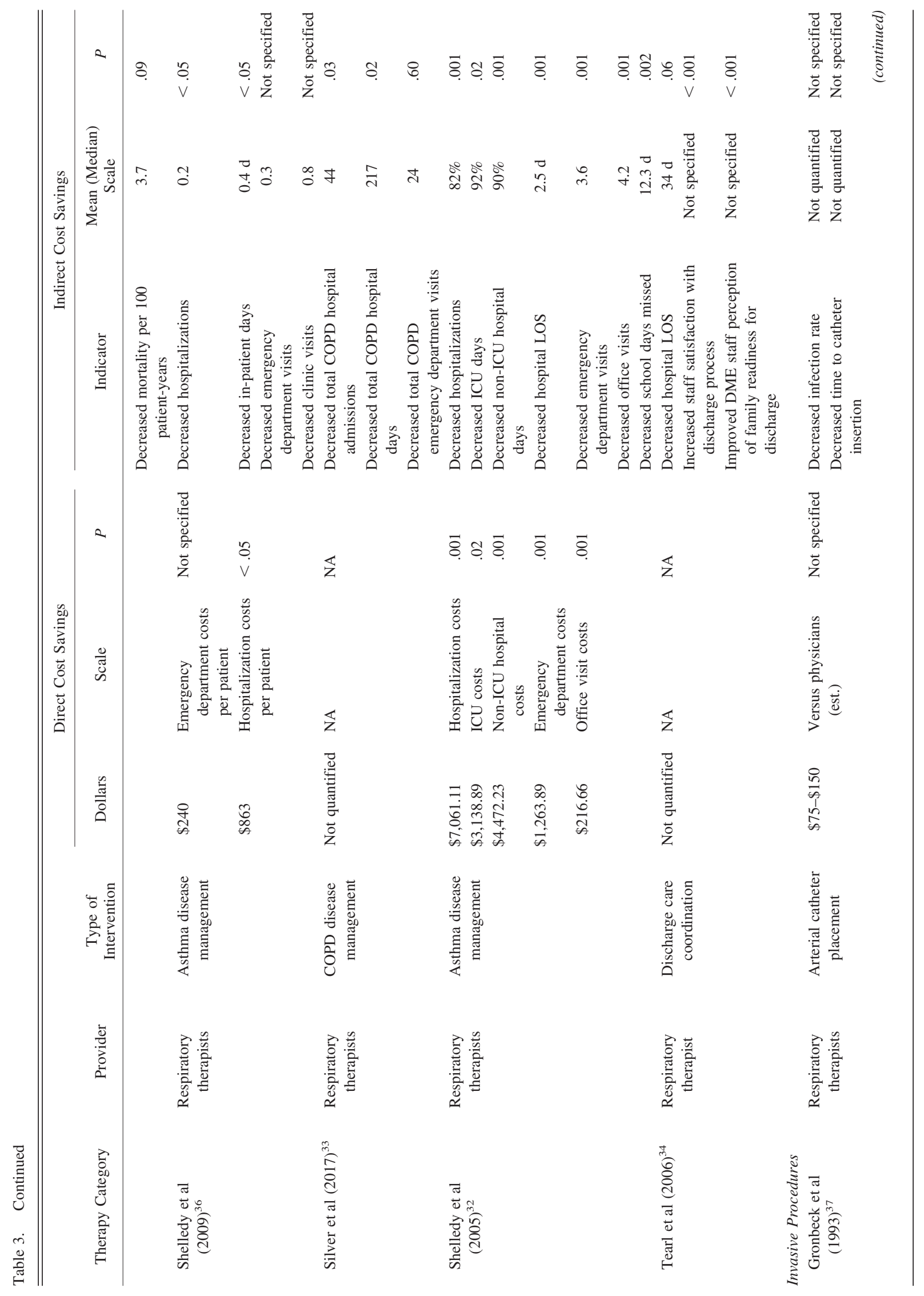


Using RTs to Reduce Cost of CARE

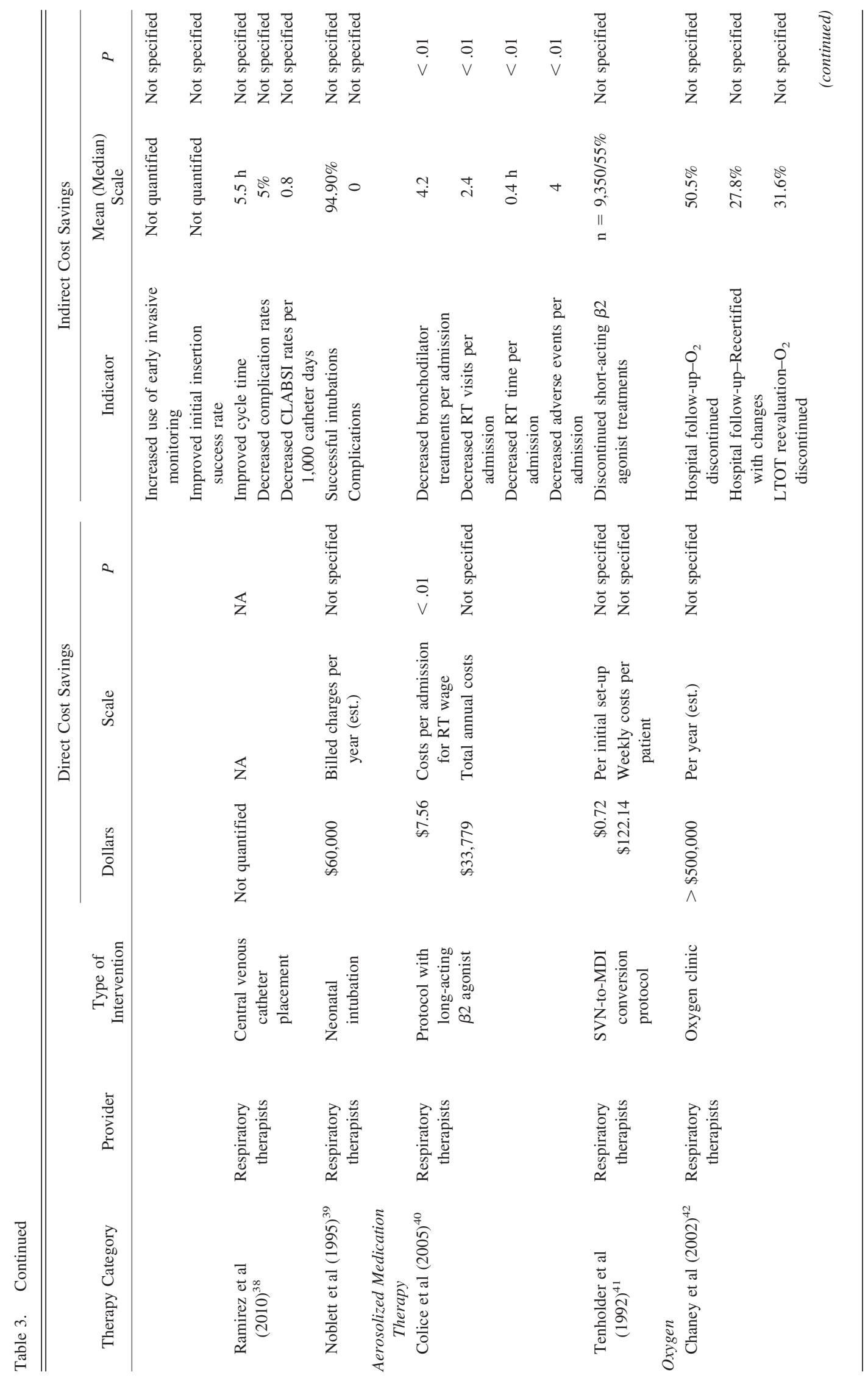


Using RTs to Reduce Cost of Care

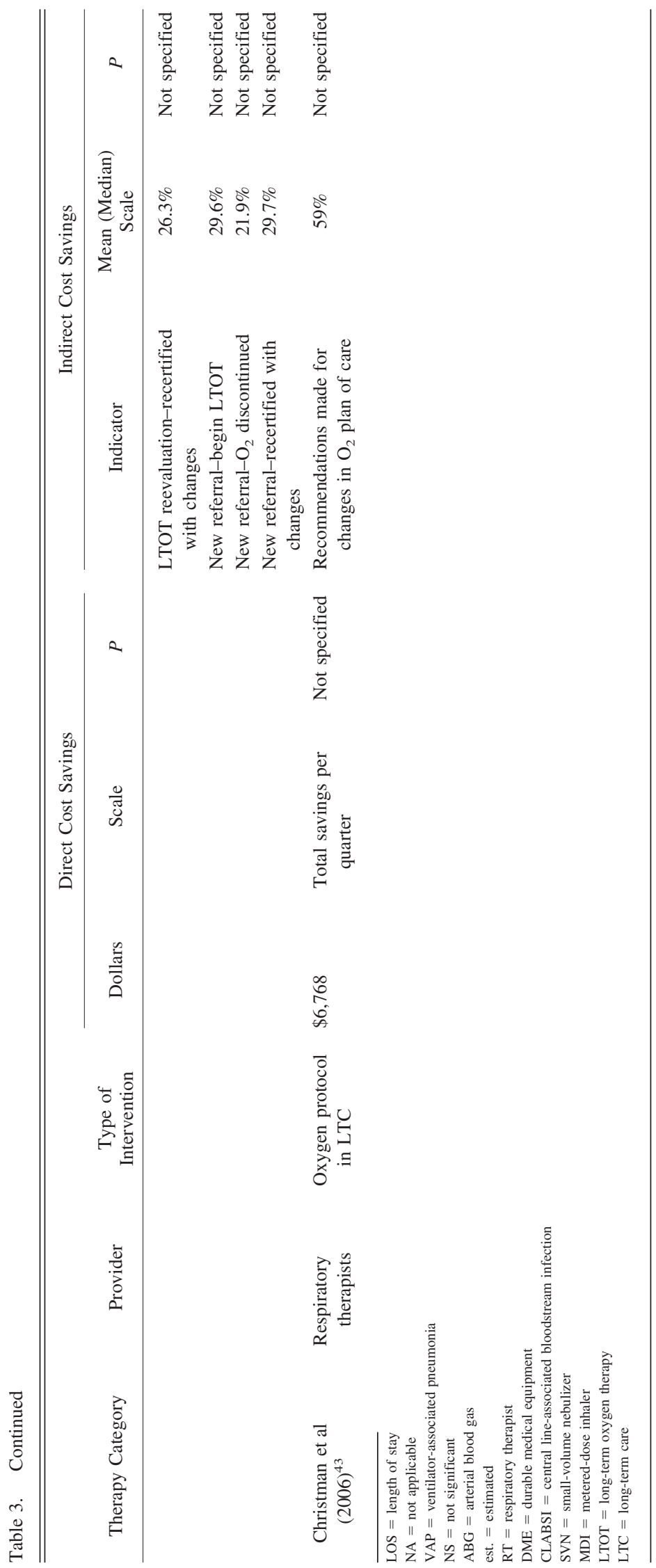


disease management, and oxygen therapy, and studies that included multiple therapies indicate that protocols span a broad range of respiratory care practices. In total, 16 studies included the use of protocol-directed care. Of these 16 studies, 6 reported a statistically significant reduction in direct costs. Of those 6 studies, $83 \%$ documented a cost savings when the protocols were directed by respiratory therapists. Four studies $25,27,31,40$ compared protocoldirected care by a RT to traditional delivery of care by a physician. In all 4 studies, it was documented that the RT could deliver more cost-effective care than their physician counterparts without any documented compromise in quality.

\section{Discussion}

The randomized controlled trial study design provides the strongest level of evidence. However, only $21 \%$ of articles utilized this design. The most popular study design was the non-concurrent cohort design. This approach is consistent with quality improvement projects that may be conducted in local departments. Although the information learned from quality improvement projects is important to note trends worthy of further study, the rigor of more scientific study is needed to confirm the findings. The findings from the current review are similar to those of a systematic review in 2000 where there was also a call for more randomized controlled trials and studies using comparison groups. ${ }^{46}$ The passage of nearly two decades yielded little change in the frequency of published randomized controlled trials using comparison groups.

One change that occurred over the span of the last 16 years was the publication of more disease-management articles. Opportunities also exist to save costs in preventing readmissions or providing care in the home. To some extent, the 5 articles that fell into the category of disease management relate to preventing hospital and emergency department admissions, as well as office visits. Disease management initiatives fit well with the vision of our future health care model. As we transition from a hospital-based provider-centric model of care to a community-based, patient-centric model, these types of studies and the evidence they develop will provide the respiratory care profession with a roadmap to shift its primary focus to collaborative, coordinated care across the continuum. It is important for the expertise of RTs to be integrated into the case-management team as their recommendations were often not included in the past.

\section{Professional Needs}

The author list from this review highlights a few themes related to researchers who publish in this area. Three authors led the publication of $25 \%$ of the articles. Three of the articles (11\%) had no RT in the authorship list. While RTs were lead authors in only 8 (29\%) publications (MDs were lead authors in 64\%), RTs did appear in the authorship list in $79 \%$ of the articles. This speaks to a present concern within the profession that only a limited number of RTs publish research. Although students are exposed to research skills in undergraduate programs, designing and executing a research project occurs at the master's degree level. ${ }^{47}$ Doctoral programs either prepare graduates for professional training (ie, MD) or for research (ie, $\mathrm{PhD}$ ). The goal for $\mathrm{PhD}$ graduates is to make original contributions to knowledge and thus become independent researchers. ${ }^{48}$ The Committee on the Accreditation for Respiratory Care reported that in $2014,88.4 \%$ of graduates held the associate degree. ${ }^{49}$ The pathway to a doctoral degree program is longer for these graduates than those with bachelor's degrees. As a result, the number of clinicians who will likely lead research initiatives is naturally less in the respiratory care profession. All but one of studies in this systematic review had at least one author with a doctoral degree. Authors with medical doctorates (26 studies) were most commonly represented. Notably, 2 RTs with $\mathrm{PhD}$ degrees were represented in the author list (3 studies). Increasing the number of RTs with doctoral degrees is needed to increase publication rates related to respiratory care.

One other professional need is related to the use of protocols. Respiratory therapists who utilize protocols can practice at the top of their license. Within stated parameters, they assess patients, recommend treatments, and evaluate the effectiveness of therapies in contrast to simply implementing a treatment plan prescribed by a medical provider. The positive outcomes of therapist-driven protocols can also enhance therapists' job satisfaction. A recent study involving RTs in the state of New York demonstrated that $22 \%$ of therapists indicated that the most important aspect of employment was their scope of practice or room for growth. The same study indicated that the most important incentive for retention was the development of a career ladder followed by increased scope of practice. Therapists who planned to leave the profession cited retirement, limited ability for growth or scope of practice and the lack of confidence in the profession's future..$^{50}$ The most common professions they transitioned to were nursing (33\%) and physician assistant (37\%). The use of RT protocols not only provides cost savings but also have the potential to retain engaged RTs in the profession.

Among the most striking findings is the variety of ways that data were reported. Notwithstanding the different study designs, the primary outcome variables for similar topics also differed. These differences make clear comparisons among studies difficult and limit the ability to compute effect sizes and appropriately explore the topic more deeply in future studies. 
Table 4. Recommendations to Facilitate Comparisons in Future Cost-Savings Studies

\begin{tabular}{|c|c|}
\hline Topic & Recommendations \\
\hline 1. Standardized units of measure & $\begin{array}{l}\text { - Cost savings: Report as dollars saved per patient, per admission or per episode of care. } \\
\text { - Reductions in length of stay: Utilize the measure "days" and report to the tenth decimal point. Examples are: } \\
\text { o decreased ventilator days } \\
\text { o decreased hospital days } \\
\text { o decreased ICU days } \\
\text { o decreased non-ICU days } \\
\text { - Resource utilization: Break down into the following categories: } \\
\text { - equipment dollars saved } \\
\text { o medication dollars saved } \\
\circ \text { salary dollars saved }\end{array}$ \\
\hline 2. Study design & - Utilize robust study designs such as randomized controlled trials. \\
\hline 3. Future research/funding & $\begin{array}{l}\text { - Prioritize funding for the following patient care areas: } \\
\text { o post-acute care } \\
\text { ○ home care } \\
\text { - Include a comparison group of other health professionals. }\end{array}$ \\
\hline 4. Establish research network & - Fund experienced researchers to mentor junior researchers from the design through publication phases. \\
\hline
\end{tabular}

\section{Recommendations}

There are several recommendations to strengthen future research initiatives and more clearly identify the value of respiratory therapists. We found it difficult to make comparisons among studies due to the wide variety of units to report outcome measures. The respiratory care profession needs standardized reporting measures so that findings from studies can be compared to one another clearly. These standardized measures should specify the values to be reported in both the numerator and the denominator of study outcome variables, units of measure, and recommended validated measures. A uniform reporting measure would help researchers compute effect sizes from studies more easily, and the knowledge gained could advance respiratory care more effectively. Suggestions for standardized outcome units include reporting all cost savings in dollars saved per patient per admission or episode of care, reporting length of stay savings in total days (to the tenth decimal), and reporting estimated cost savings associated with decreased length of stay in terms of decreased ventilator days, decreased hospital days, decreased ICU days, and decreased non-ICU days. Much of this information should be readily available from hospital finance departments. We also suggest that resource-utilization cost savings should be broken down by equipment, medication, and salary (not hours saved). An example of a useful compensation calculator can be found in the most recent AARC Human Resource Survey (http://www.aarc.org/resources/ tools-software/aarc-respiratory-therapist-human-resourcestudy-2014 Accessed November 9, 2017) for the appropriate geographical region under study to determine the predicted salary savings.

The current findings highlight the areas that have a solid base of literature and where gaps are present. One area alluded to earlier was the limited number of studies that utilized randomized controlled trial designs. Two areas of practice where research on the value of the RT is extremely limited are post-acute care and home-care settings. Funding agencies may want to consider prioritizing research funding to highlight these areas because many of the recent health care reform initiatives are focused on patient care outside of the acute care areas.

It would also be valuable for the respiratory care profession to establish a research network. For topic areas where there is a high need for replication studies, funding from a national organization such as the American Respiratory Care Foundation could be established for experienced researchers to provide consultation with emerging researchers from the design phase of the research project throughout the reporting phase. Less experienced researchers often delay requesting help until the analysis phase of their projects. Because the design and data collection are critical to study integrity, providing resource help at the start of projects for high-need areas has a greater chance of providing more robust outcomes and will also enhance the skill set of junior researchers.

We also recommend that future studies should have a comparison group if the value of the RT is the outcome variable. There were several studies that evaluated the impact of changes in respiratory care practice, but they were excluded from this study because they concentrated on the benefits of a specific piece of equipment and did not demonstrate the specific value added by the RT. These recommendations are summarized in Table 4.

\section{Limitations}

This study had several limitations. The list of search terms may have been incomplete and may have resulted in 
missed articles. Some articles retrieved in the search may have been excluded erroneously due to the relatively large number of resulting articles. The varied methods of reporting costs and the broad range of respiratory care described prevented us from conducting a meta-analysis. In addition, the number of studies in each topic area except for mechanical ventilation was small, so the findings across a greater number of similar studies could yield different results as respiratory care practices may not be the same in each health care system. Lastly, researchers are more likely to report and journal editors more likely to publish studies with positive results. The absence of negative studies could have skewed the reported findings.

\section{Conclusions}

Across a broad range of respiratory care topics, all reported studies demonstrated cost savings in subjects who suffer from cardiopulmonary disease. These cost savings were achieved through RT-driven protocols, utilization of the unique expertise of the RTs in patient assessment, autonomous decision making, and the ability to independently perform invasive procedures. However, there was high variability in the study designs, settings, and topics addressed, which prevented a detailed comparative analysis of cost savings. The areas with the best quality of research was mechanical ventilation and disease management, and there was a complete absence of research in home care. These findings should be used to direct future respiratory care research priorities. A mentoring network is needed to facilitate an increase in high-quality respiratory care studies.

\section{REFERENCES}

1. Smith, GA. Respiratory care: evolution of a profession. Olathe, KS: Applied Measurement Professionals; 1989.

2. Ward JJ, Helmholz HF. Roots of the respiratory care profession. In: Burton GG, Hodgkin JE, Ward JJ. Respiratory care: a guide to clinical practice, 4th ed. Philadelphia, PA: Lippincott Williams \& Wilkins; 1997:3-25.

3. Murray JF. Review of the state of the art in intermittent positive pressure breathing therapy. Am Rev Respir Dis 1974;110(6 Pt 2): 193-199.

4. Restrepo RD, Wettstein R, Wittnebel L, Tracy M. Incentive spirometry: 2011. Respir Care 2011;56(10):1600-1604.

5. Strickland SL, Rubin BK, Drescher GS, Haas CF, O'Malley CA, Volsko TA, et al. AARC clinical practice guideline: effectiveness of nonpharmacologic airway clearance therapies in hospitalized patients. Respir Care 2013;58(12):2187-2193.

6. Strickland SL, Rubin BK, Haas CF, Volsko TA, Drescher GS, O'Malley CA. AARC clinical practice guideline: effectiveness of pharmacologic airway clearance therapies in hospitalized patients. Respir Care 2015;60(7):1071-1077.

7. Miller HD. From volume to value: better ways to pay for health care. Health Aff (Millwood) 2009;28(5):1418-1428.

8. Fink JB, Fink AK. The respiratory therapist as manager. Chicago, IL: Year Book Medical Publishers; 1986.
9. Catlin AC, Cowan CA. History of health spending in the United States, 1960-2013. Baltimore, MD: Centers for Medicare and Medicaid Services; 2015.

10. Nielsen-Tietsort J, Poole B, Creagh CE, Repsher LE. Respiratory care protocol: an approach to in-hospital respiratory therapy. Respir Care 1981;26(5):430-436.

11. Rosenthal MB. Beyond pay for performance: emerging models of provider-payment reform. N Engl J Med 2008;359(12):1197-200.

12. Centers for Medicare and Medicaid Services. Roadmap for implementing value driven healthcare in the traditional Medicare fee-forservice program. Baltimore, MD: Department of Health and Human Services; 2009.

13. Berwick DM, Nolan TW, Whittington J. The triple aim: care, health, and cost. Health Aff (Millwood) 2008;27(3):759-769.

14. Moher D, Liberati A, Tetzlaff J, Altman DG, Prisma Group. Preferred reporting items for systematic reviews and meta-analyses: the PRISMA statement. PLoS Med 2009;6(7):e1000097.

15. Viswanathan M, Berkman ND, Dryden DM, Hartling L. Assessing risk of bias and confounding in observational studies of interventions or exposures: further development of the RTI Item Bank. Rockville, MD: Agency for Healthcare Research and Quality (US); 2013, Report No. 13-EHC106-EF.

16. Burns SM, Earven S, Fisher C, Lewis R, Merrell P, Schubart JR, et al. Implementation of an institutional program to improve clinical and financial outcomes of mechanically ventilated patients: one-year outcomes and lessons learned. Crit Care Med 2003;31(12):27522763.

17. Ely EW, Baker AM, Dunagan DP, Burke HL, Smith AC, Kelly PT, et al. Effect on the duration of mechanical ventilation of identifying patients capable of breathing spontaneously. N Engl J Med 1996; 335(25):1864-1869.

18. Gupta P, Giehler K, Walters RW, Meyerink K, Modrykamien AM. The effect of a mechanical ventilation discontinuation protocol in patients with simple and difficult weaning: impact on clinical outcomes. Respir Care 2014;59(2):170-177.

19. Kelleghan SI, Salemi C, Padilla S, McCord M, Mermilliod G, Canola $\mathrm{T}$, et al. An effective continuous quality improvement approach to the prevention of ventilator-associated pneumonia. Am J Infect Control 1993;21(6):322-330.

20. Kollef MH, Shapiro SD, Silver P, St John RE, Prentice D, Sauer S, et al. A randomized, controlled trial of protocol-directed versus physician-directed weaning from mechanical ventilation. Crit Care Med 1997;25(4):567-574.

21. Dasgupta A, Rice R, Mascha E, Litaker D, Stoller JK. Four-year experience with a unit for long-term ventilation (respiratory special care unit) at the Cleveland Clinic Foundation. Chest 1999;116(2): 447-455.

22. Cohen IL, Bari N, Strosberg MA, Weinberg PF, Wacksman RM, Millstein $\mathrm{BH}$, et al. Reduction of duration and cost of mechanical ventilation in an intensive care unit by use of a ventilatory management team. Crit Care Med 1991;19(10):1278-1284.

23. Hermeto F, Bottino MN, Vaillancourt K, Sant'Anna GM. Implementation of a respiratory therapist-driven protocol for neonatal ventilation: impact on the premature population. Pediatrics 2009;123(5): e907-e916.

24. Arroliga AC, Pollard CL, Wilde CD, Pellizzari SJ, Chebbo A, Song $\mathrm{J}$, et al. Reduction in the incidence of ventilator-associated pneumonia: a multidisciplinary approach. Respir Care 2012;57(5):688-696.

25. Kallam A, Meyerink K, Modrykamien AM. Physician-ordered aerosol therapy versus respiratory therapist-driven aerosol protocol: the effect on resource utilization. Respir Care 2013;58(3):431-437.

26. Kollef MH, Shapiro SD, Clinkscale D, Cracchiolo L, Clayton D, Wilner R, et al. The effect of respiratory therapist-initiated treatment 


\section{Using RTs to Reduce Cost of CARE}

protocols on patient outcomes and resource utilization. Chest 2000; 117(2):467-475.

27. Harbrecht BG, Delgado E, Tuttle RP, Cohen-Melamed MH, Saul MI, Valenta CA. Improved outcomes with routine respiratory therapist evaluation of non-intensive-care-unit surgery patients. Respir Care 2009;54(7):861-867.

28. Werre ND, Boucher EL, Beachey WD. Comparison of therapistdirected and physician-directed respiratory care in COPD subjects with acute pneumonia. Respir Care 2015;60(2):151-154.

29. Nyland BA, Spilman SK, Halub ME, Lamb KD, Jackson JA, Oetting $\mathrm{TW}$, et al. A preventative respiratory protocol to identify trauma subjects at risk for respiratory compromise on a general in-patient ward. Respir Care 2016;61(12):1580-1587.

30. Stoller JK, Mascha EJ, Kester L, Haney D. Randomized controlled trial of physician-directed versus respiratory therapy consult servicedirected respiratory care to adult non-ICU inpatients. Am J Respir Crit Care Med 1998;158(4):1068-1075.

31. Stoller JK, Skibinski CI, Giles DK, Kester EL, Haney DJ. Physicianordered respiratory care vs physician-ordered use of a respiratory therapy consult service: results of a prospective observational study. Chest 1996;110(2):422-429.

32. Shelledy DC, McCormick SR, LeGrand TS, Cardenas J, Peters JI. The effect of a pediatric asthma management program provided by respiratory therapists on patient outcomes and cost. Heart Lung 2005; 34(6):423-428.

33. Silver PC, Kollef MH, Clinkscale D, Watts P, Kidder R, Eads B, et al. A respiratory therapist disease management program for subjects hospitalized with COPD. Respir Care 2017;62(1):1-9.

34. Tearl DK, Cox TJ, Hertzog JH. Hospital discharge of respiratorytechnology-dependent children: role of a dedicated respiratory care discharge coordinator. Respir Care 2006;51(7):744-749.

35. Rice KL, Dewan N, Bloomfield HE, Grill J, Schult TM, Nelson DB, et al. Disease management program for chronic obstructive pulmonary disease: a randomized controlled trial. Am J Respir Crit Care Med 2010;182(7):890-896.

36. Shelledy DC, Legrand TS, Gardner DD, Peters JI. A randomized controlled study to evaluate the role of an in-home asthma disease management program provided by respiratory therapists in improving outcomes and reducing the cost of care. J Asthma 2009;46(2): 194-201.
37. Gronbeck C 3rd, Miller EL. Nonphysician placement of arterial catheters: experience with 500 insertions. Chest 1993;104(6):1716-1717.

38. Ramirez C, Malloch K, Agee C. Evaluation of respiratory care practitioner central venous catheter insertion program. The Journal of the Association for Vascular Access 2010;15(4):207-211.

39. Noblett KE, Meibalane R. Respiratory care practitioners as primary providers of neonatal intubation in a community hospital: an analysis. Respir Care 1995;40(10):1063-1067.

40. Colice GL, Carnathan B, Sung J, Paramore LC. A respiratory therapist-directed protocol for managing inpatients with asthma and COPD incorporating a long-acting bronchodilator. J Asthma 2005; 42(1):29-34.

41. Tenholder MF, Bryson MJ, Whitlock WL. A model for conversion from small volume nebulizer to metered dose inhaler aerosol therapy. Chest 1992;101(3):634-637.

42. Chaney JC, Jones K, Grathwohl K, Olivier KN. Implementation of an oxygen therapy clinic to manage users of long-term oxygen therapy. Chest 2002;122(5):1661-1667.

43. Christman SL, Volsko TA. Evaluation of an oxygen protocol in long-term care. Respir Care 2006;51(12):1424-1431.

44. American Thoracic Society. Statement on home care for patients with respiratory disorders. Am J Respir Crit Care Med 2005;171(12): 1443.

45. James J. Health policy brief: Medicare hospital readmissions reduction program. Health Affairs; Nov. 12, 2013.

46. Stoller JK. 2000 Donald F. Egan scientific lecture. Are respiratory therapists effective? Assessing the evidence. Respir Care 2001;46(1): 56-66.

47. Adelman C, Ewell P, Gaston P, Schneider CG. The degree qualifications profile: a learning-centered framework for what college graduates should know and be able to do to earn the associate, bachelor's or master's degree. Indianapolis, IN: Lumina Foundation for Education; 2014

48. Council of Graduate Schools. The doctor of philosophy degree: a policy statement. Washington, D.C.; 1990.

49. Commission on Accreditation for Respiratory Care. Accreditation in respiratory care education. Bedford, TX; 2016.

50. Smith SG, Endee LM, Benz Scott LA, Linden PL. The future of respiratory care: results of a New York State survey of respiratory therapists. Respir Care 2017;62(3):279-287. 